\title{
Books Received at the Editorial Office
}

Appearance of a title in this list does not preclude the possibility that a review may appear in a later issue.

H.S. Badeer: Cardiovascular Physiology. Karger Continuing Education Series, vol. 6. Karger, Basel 1984.

XVI + 276 pp.; SFr. 58.- I DM 69.- I US\$ 34.75. ISBN 3-8055-3796-4.

T. Godfrained, A.G. Herman: Calcium Entry Blockers in Cardiovascular and Cerebral Dysfunctions. Nijhoff, The Hague 1984. 330 pp.; Dfl. 135.-/US\$ 55.00. ISBN 0-89838-658-6. L. David Hillis, B.G. Firth: Manual of Clinical Problems in Cardiology. Little, Brown, Boston 1984. 393 pp.; US\$ 17.95. ISBN 0-316-36401-0.

Carlo Marchesi, Ambulatory Monitoring. Cardiovascular System and Allied Applications. Nijhoff, The Hague 1984.

XVI + 420 pp.; Dfl. 170.-/US\$ 65.00. ISBN 0-89838-642-X.

St. W. Miller: Cardiac Angiography. Little, Brown, Boston 1984. XII + 430 pp.; US\$ 76.50. ISBN 0-316-57367-1.

J. Morganroth, E.N. Moore: Interventions in the Acute Phase of Myocardial Infarction. Nijhoff, The Hague 1984.

XII + 320 pp.; Dfl. 134.-/US\$ 39.95. ISBN 0-89838-659^*.

W.C. Randall: Nervous Control of Cardiovascular Function. Oxford University Press, Oxford 1984. XIV + 476 pp.; E 35.-. ISBN 0-19-503390-6.

D.C. Rao, Robert C. Elston: Genetic Epidemiology of Coronary Heart Disease. Progress in Clinical and Biological Research, vol. 147. Liss, New York 1984. XXIII + 575 pp.; E 60.-. ISBN 0-8451-0147-1.

R.H. Swanton: Cardiology. Blackwell, Oxford 1984. 438 pp.; E 8.50. ISBN 0-632-01099-1. G.C. Timmis: Cardiovascular Review 1984. Academic Press, London 1984. XXXVII + 705 pp.; US\$ 44.00. ISBN 0-12-691346-3.

Paul N. Yu, John F. Goodwin: Progress in Cardiology. Lea \& Febiger, Philadelphia 1983. XVII+ 290 pp.; US\$49.50. ISBN 0-8121-0911-2.

Biological Effects of Ultrasound: Mechanisms and Clinical Implications. NCRP Report, No. 74. National Council on Radiation Protection and Measurements, Bethesda 1983. XIII + 266 pp. ISBN 0-913392-64-2. 